\title{
Influence of Sustained Beta-Alanine Supplementation on Body Composition and Physical Performance in College-aged Males Seeking Military Commission
}

\author{
Daniel Jaffe ${ }^{1, *}$, Jennifer Hewit ${ }^{1}$, Jason Cholewa ${ }^{2}$, Alexander Bedard ${ }^{1}$, Tracey Matthews ${ }^{3}$, \\ Brian Thompson ${ }^{3}$, Samuel Headley ${ }^{3}$ \\ ${ }^{1}$ Department of Physical Education, United States Military Academy, United States \\ ${ }^{2}$ Department of Kinesiology, Coastal Carolina University, United States \\ ${ }^{3}$ Department of Exercise Science and Sport Studies, Springfield College, United States
}

Copyright $\bigcirc 2018$ by authors, all rights reserved. Authors agree that this article remains permanently open access under the terms of the Creative Commons Attribution License 4.0 International License

\begin{abstract}
The present study was designed to investigate the effects of chronic ingestion of a proprietary beta-alanine supplement on body composition, indices of strength and power performance, and markers of cardiovascular endurance in physically active, college-aged males commissioning within the armed services. Subjects $(\mathrm{n}=30)$ participated in four separate testing sessions, both pre and post assessment consuming either $6.0 \mathrm{~g} /$ day beta-alanine or Placebo supplementation for 6 weeks. Parameters evaluated included body fat percentage (BF\%), fat free mass (FFM), vertical jump (VJ), 1 repetition maximum back squat (SQ) and bench press (BP), and a repetition maximum bench press (RMBP) at $75 \%$ of the attained maximum, maximal oxygen consumption $\left(\mathrm{VO}_{2 \text { peak }}\right)$, respiratory exchange ratio (RER), lactate threshold (LT), and peak lactate concentration. For all variables, no significant interactions existed across condition and time $(p>.05)$. In addition, for all variables, no significant differences existed between conditions $(p>.05)$. Beta-alanine did not show any benefit for improving body composition or physical performance parameters in this group of individuals.
\end{abstract}

Keywords Beta Alanine, Strength, Endurance, Military

\section{Introduction}

Increases in muscular strength have been shown to correlate with improved sprinting speed, jumping performance, and ease of performing activities of daily living $[1,2]$. Additionally, improvements in body composition have been shown to directly correlate with athletic performance across a multitude of activities $[3,4$,
5]. As the ability to increase lean mass diminishes progressively with training experience, many athletes employ the use of nutritional supplements or ergogenic aids to aid in facilitating muscle and strength gains $[6,7]$.

Homeostasis is defined as the maintenance of a constant internal environment, with exercise being a planned deviation from this consistency [8]. A decline in $\mathrm{pH}$, attributed to the liberation of lactate, hydrogen ions and the formation of carbon dioxide as a consequence of energy production, presents itself as a complex threat to homeostasis that must be managed at both the extracellular and intracellular levels [8]. In the extracellular environment red blood cells (RBCs) have two primary functions within the body: delivery of oxygen from the lungs to the metabolically active tissues, and uptake of carbon dioxide from these metabolically active tissues for unloading in the lungs [9]. These RBCs contain molecules of hemoglobin, responsible for carrying and transporting oxygen in the blood to the metabolically active tissues [9]. One molecule of hemoglobin contains four protein chains, called globins, and a non-protein molecule, or pigment, called the heme group. The heme group, which contains iron, is responsible for binding free oxygen for transport and delivery [9].

Carbon dioxide on the other hand, is typically transported in the plasma in the form of a bicarbonate ion $[10,9]$. This bicarbonate ion, which picks up liberated hydrogen ions forming carbonic acid, is then transported to the lungs. Once in the lungs, the carbonic acid is broken down, forming carbon dioxide and water, which is then expired [11]. The lactate ion can then be transported to the liver and reconverted to glucose and stored as glycogen for later use [8].

The intracellular environment provides a fairly different picture. During high intensity training, anaerobic glycolysis is the dominant system of energy production 
[12]. Examples of activities that rely predominantly on anaerobic glycolysis for energy would be activities of short duration, ranging from the $100-800 \mathrm{~m}$ run in track $[13,14]$, Greco-Roman wrestling [15], and certain military fitness assessments across all branches of the services $[16,17,18$, 19]. Researchers have also demonstrated the role played by glycolysis and subsequent lactate formation and accumulation as a means of predicting performance for such long duration activities as the $3,000 \mathrm{~m}$ and up to the $10,000 \mathrm{~m}$ run [20].

Anaerobic glycolysis involves the rapid breakdown of glucose into lactate within the cytosol of the cell. This lactate production results in the dissociation of hydrogen ions accounting for the generation of more than $94 \%$ of the total hydrogen ion concentration within skeletal muscle, causing a dramatic decline in intramuscular $\mathrm{pH}[12,21]$. This reduction in $\mathrm{pH}$ will eventually overwhelm the cell's buffering capacity, resulting in acidosis, hindering the ability of the muscle to continue to contract, resulting in muscular fatigue and a decline in performance [21]. Physiochemical buffers within the cell, specifically carnosine, provide the primary mechanism for buffering these liberated ions, delaying the onset of acidosis and aiding in performance $[22,23]$.

Diet and muscle fiber type are all major factors in predicting intramuscular carnosine concentration [22]. Constituent substrate availability, including the amino acids L-histidine and more specifically beta-alanine, play the greatest role in determining the concentration of intramuscular carnosine [22]. Beta-alanine has been identified by many researchers as the rate-limiting factor in intramuscular carnosine concentration, meaning that without this beta-amino acid, individuals would be deficient in carnosine and in turn they would no longer be able to adequately maintain homeostasis at the intracellular level during physical exertion [23, 22, 17, 18, 24]. Researchers therefore have supported the notion that extraneous beta-alanine supplementation would augment carnosine synthesis, allowing individuals to better buffer intracellular hydrogen ions, permitting sustained efforts for greater periods of time at higher exercise intensities [23, 18]. Specifically, beta alanine ingestion may enhance performance of activities relying heavily on anaerobic glycolysis [13, 16, 14, 15, 21, 19].

\section{Objectives}

The primary purpose of this research study was to investigate the effects of chronic ingestion of a proprietary beta alanine supplement on body composition, indices of strength and power performance, and markers of cardiovascular endurance in physically active, college-aged males commissioning within the Air Force, Army, Navy and Marine Corps. As performance on these physical tests often means the difference between being offered a commission and being rejected from the selected branch of service, any potential advantage gained by supplementation could play a major role in determining the future of these cadets. The researcher hypothesized that chronic supplement ingestion would result in improved body composition, enhanced indices of strength and power performance, and improvements in markers of cardiovascular endurance, and a delay in the onset of blood lactate accumulation, specifically the lactate threshold (LT).

\section{Methods}

The present study was designed with the goal of identifying the effects of 6 weeks of beta alanine supplementation on body composition, indices of strength and power performance, and markers of cardiovascular endurance in college-aged males.

\subsection{Subjects}

The subjects in the present investigation were 30 physically active, resistance trained males. Subjects were between the ages of 18-22 years with an average body mass of between 150-180 pounds, currently attending a small state college in the Southwestern Virginia area. Subjects were randomly assigned into two groups. Prior to participating in the investigation, subjects read and signed a consent form, completed a medical history questionnaire, as well as documentation of training history. Subjects were asked to refrain from intense exercise $24 \mathrm{hr}$ prior to testing. Additionally, subjects were asked to refrain from using any additional dietary supplements throughout the duration of the investigation. Upon completion of all testing, each of the subjects was provided with the results of their body composition analysis and exercise performance tests.

\section{Instrumentation}

The instrumentation required to perform the investigation included: Air displacement plethysmography (BOD POD GS, COSMED Pulmonary Function Equipment, Chicago, IL) to estimate body mass and body composition; a "Just-Jump" mat (Just-Jump!, Probotics Inc., Huntsville, AL) to determine vertical jump height; a power rack with barbell and sufficient weight (Power Systems, Knoxville, TN) to perform strength testing assessments; a cycle ergometer (VIAsprint ${ }^{\mathrm{TM}}$ 150P Bicycle, CareFusion, San Diego, CA) for conducting cardiorespiratory endurance tests; a metabolic cart (Vmax ${ }^{\mathrm{TM}}$ Encore Metabolic Cart, CareFusion, San Diego, CA) for quantifying cardiorespiratory endurance tests; and a lactate meter (Accutrend $₫$ Lactate, Roche, New York, NY) for identifying the lactate threshold and peak lactate concentration. 


\subsubsection{Diet Log}

Each subject was instructed to complete a 3-day diet log of their habitual diet prior to initiation of the experimental testing sessions. Subjects were asked to keep a copy of this diet $\log$ and replicate their initial dietary intake for the 3 days prior to the second round of testing to ensure consistency. The subjects were asked to maintain a relatively consistent caloric intake throughout the duration of the intervention. The risk of extraneous influence on blood parameters is significantly reduced by consuming the same diet prior to each testing session [23].

\subsubsection{Proprietary Nutritional Supplement}

Subjects were randomly assigned to either an experimental or a placebo group. Subjects consumed a prescribed dosage of either the dietary supplement or an isocaloric, taste-matched placebo for the duration of the intervention in an independent groups design.

The supplement was composed of beta-alanine and maltodextrin. Each of these ingredients was measured out by a third party to ensure that neither the subject nor experimenter knew which supplement had been administered. The quantity of each ingredient in the supplement was selected based upon the amount used by Jordan et al. [24]. The placebo supplement was of identical flavor and contained a quantity of maltodextrin equivalent to the relative quantity of aforementioned ingredients [24].

\subsection{Testing Protocol}

Each subject met with the primary investigator on four separate occasions. All paperwork was completed along with body composition and indices of cardiovascular fitness during the first session. The second session consisted of the evaluation of power, strength, and muscular endurance. Sessions three and four were identical to the procedures followed during sessions one and two.

For session one, subjects arrived at the human physiology lab following an 8-hr fast. Subjects filled out all necessary paperwork. A final evacuation occurred, with hydration being assessed via visual urinalysis [25]. Body composition was then estimated. Subjects were fitted with appropriate head gear, mouth piece and heart rate monitor to begin the VO2Peak test. Following a 3 min rest period, initial capillary puncture was taken to identify baseline lactate levels. All subjects performed the McArdle graded cycling protocol for determining VO2Peak [26]. Capillary puncture occurred during the final $30 \mathrm{sec}$ of each stage to track changes in blood lactate concentration. This occurred following heart rate and rate of perceived exertion (RPE) assessment. The lactate threshold (LT) was identified as a blood concentration of lactate in excess of $4.0 \mathrm{mmol} / \mathrm{L}$ [24]. Testing stopped once subjects reached volitional fatigue or met the criteria to qualify assessment as a VO2Peak test, including an RPE of 10, a plateau in maximal oxygen uptake despite an increase in workload, and a heart rate within 10 beats per minute of the age predicted maximum [27]. Vital signs and blood lactate were measured at $5 \mathrm{~min}$ post exercise cessation. Upon completion of cardiovascular testing, subjects were provided a meal replacement bar (Met-RX Big 100, Met-RX Substrate Technology, Inc., Bohemia, NY) then instructed on how to keep a diet log, incorporating a dietary recall of the 72 hours preceding initial testing and an additional 72 hour period prior to final testing.

Session two took place $24 \mathrm{hr}$ after initiation of the VO2Peak test. Following a minimum 2-hr fast, subjects followed a prescribed warm-up of dynamic stretches. Subjects were instructed as to how to perform each of these stretches by a Certified Strength and Conditioning Specialist (CSCS). During all testing sessions, subjects were permitted to consume water Ad Libitum. Following the designated warm-up, subjects began the testing procedure.

The first test administered was the countermovement vertical jump (VJ) test. The VJ test assessed muscular power. Each subject performed three countermovement VJs, separated by $2 \mathrm{~min}$ of rest. During the countermovement VJ, each subject was instructed to start in a stationary position. The downward phase of the jump consisted of the subjects flexing at the hips and knees to approximately $90^{\circ}$, with concurrent shoulder hyperextension. The upward phase of the countermovement VJ consisted of concurrent rapid hyperextension of the knee and hip, and flexion of the shoulder. Arm swing was permitted during performance of the VJ. VJ height was recorded using a "Just-Jump" mat. The maximum height achieved during the three trials was recorded and used for data analysis.

The one-repetition maximum (RM) for both the back squat (SQ) and the bench press (BP) was determined next using the method described by Baechle, Earle, and Wathen [28]. After completion of three warm-up sets, the weight for each of the exercises was subsequently increased for each subject until a one-repetition maximum was performed before exhaustion. The rest between sets was 2-4 min in length, as outlined by Baechle et al. [28]. Upon completion of 1-RM testing, the subjects then performed a maximum repetition BP (RMBP) using $75 \%$, or the estimated $10 \mathrm{RM}$ amount of weight, as determined via formula [28]. Once all testing was completed, subjects were allowed to cool-down for $5 \mathrm{~min}$ at a volitional pace on a cycle ergometer.

Treatments were administered double blind and consisted of either a placebo (maltodextrin) or beta alanine (Bulk Supplements, Las Vegas, NV). The composition of the supplements was kept secret until all data had been collected. A third party designated by the primary investigator filled identical, unmarked gelatin capsules with either $0.75 \mathrm{~g}$ maltodextrin or $0.75 \mathrm{~g}$ beta alanine. Subjects consumed two capsules $(1.50 \mathrm{~g})$ four times per day yielding an absolute total of $6.0 \mathrm{~g}$ beta alanine. This 
dosage was chosen based upon data demonstrating safe dietary intakes of up to $8.0 \mathrm{~g}$ distributed evenly throughout the day [29] with the majority of the research demonstrating significant effects with a dosage between 3.2-6.4 g/day [24]. An absolute, rather than a relative quantity, was utilized in compliance with previous research and for the sake of practicality. Subjects met with the principle investigator every week to check-in and collect the 7 day supply of the designated intervention, whether the supplement or the placebo.

Session three occurred 6 weeks after session two. The protocol was identical to that followed during the first session. Session four occurred the following day and was identical to the procedure followed during session two.

\subsection{Statistical Analysis}

Two, 2 x 2 Mixed Factorial ANOVAs were used to determine the difference between groups (placebo and beta alanine) and time (pre-intervention, post-intervention) for body fat percentage and fat free mass. Four, $2 \times 2$ Mixed Factorial ANOVAs were used to determine the difference between groups (placebo and beta alanine) and time (pre-intervention, post-intervention) for strength performance (Vertical Jump, 1-Repetition Maximum Squat and Bench press, and a Repetition Maximum Bench press at $75 \%$ of $1-\mathrm{RM})$. Four, 2 × 2 Mixed Factorial ANOVAs were used to determine the difference between groups (placebo and beta alanine) and time (pre-intervention, post-intervention) for cardiovascular parameters (VO2peak, Respiratory Exchange Ratio, VE/VO2, and VE/VCO2). Finally, two $2 \times 2$ Mixed Factorial ANOVAs were used to determine the difference between groups (placebo and beta alanine) and time (pre-intervention, post-intervention) for Lactate Threshold $(4.0 \mathrm{mmol} / \mathrm{L}$ blood lactate concentration) and peak lactate concentration All statistical analyses were analyzed using Statistical Package for the Social Sciences (SPSS v. 22, IBM) with the alpha level set at .05 .

\section{Results}

Sixty subjects initially enrolled to participate in this study, with only 30 physically active, resistance-trained males completing the investigation (age $20 \pm 1.4 \mathrm{yr}$; range 18-22 yr). Reasons for withdrawal included time restrictions attributed to military obligations and other extracurricular activities. Subjects were randomly assigned to either a placebo (maltodextrin; $n=14$ ) or supplemental (beta alanine; $\mathrm{n}=16$ ) group for the duration of the six-week intervention. Each group was asked to consume $6.0 \mathrm{~g}$ of designated substrate per day, following the procedure of Hoffman et al [19]. Levene's Test of Equality of Error Variances was used to test for homogeneity of variance. Descriptive statistics for subjects are reported in Table 1.
Table 1. Descriptive Statistics for Subjects $(\mathrm{N}=30)$

\begin{tabular}{|c|c|c|c|}
\hline & Control & Supplement & Mean \pm SD \\
\hline Age (yrs) & $20.64 \pm 1.3$ & $20.31 \pm 1.4$ & $20.47 \pm 1.4$ \\
\hline Height (in.) & $67.65 \pm 4.1$ & $70.13 \pm 2.7$ & $68.97 \pm 3.6$ \\
\hline
\end{tabular}

For all variables, no significant interactions existed across condition and time. In addition, for all variables, no significant differences existed between conditions. A significant time effect was found for the following variables: $\mathrm{VE} / \mathrm{VCO}_{2}$ demonstrated higher pre-test values, while 1-RM SQ, 1-RM BP, RM BP, $\mathrm{VO}_{2 \text { Peak }}$, RER, and peak lactate concentration all demonstrated higher post-test values.

There were no significant interactions or main effects of time or group for body fat percentage $(p>.05)$. Additionally, there no significant interactions or main effects of time or group for fat free mass $(p>.05)$. Data for both body fat percentage and fat free mass are presented in Table 2.

Table 2. Body Composition for Subjects

\begin{tabular}{|c|c|c|}
\hline Time & Control & Supplement \\
\hline \multicolumn{3}{|c|}{ Body Fat Percentage (\%) } \\
\hline Pre & $15.9 \pm 5.1$ & $14.8 \pm 7.7$ \\
\hline Post & $14.9 \pm 3.7$ & $14.5 \pm 7.8$ \\
\hline \multicolumn{3}{|c|}{ Fat Free Mass (lbs) } \\
\hline Pre & $157.9 \pm 30.9$ & $151.0 \pm 26.7$ \\
\hline Post & $157.4 \pm 30.1$ & $150.9 \pm 25.6$ \\
\hline
\end{tabular}

There were no significant interactions or main effects of time or group for VJ performance $(p>.05)$. Data for VJ performance is presented in Table 3 .

There was no significant interaction or main effect of group for 1-RM SQ performance $(p>.05)$. There was a significant main effect of time for 1-RM SQ performance $(p=.003)$. The mean value for 1-RM SQ performance for both the placebo and supplemental group were significantly greater post-intervention than pre-intervention (Pre: $282.3 \pm 96.9$ lbs; Post: $297.5 \pm 102.4$ lbs). Data for 1-RM SQ performance is presented in Table 3.

There was no significant interaction or main effect of group for 1-RM BP performance $(p>.05)$. There was a significant main effect of time for 1-RM BP performance $(p=.024)$. The mean value for 1-RM BP performance for both the placebo and supplemental group were significantly greater post-intervention than pre-intervention (Pre: $216.8 \pm 61.9$ lbs; Post: $221.3 \pm 63.2$ lbs). Data for 1-RM BP performance is presented in Table 3.

There were no significant interactions or main effects of time or group for RMBP performance $(p>.05)$. Data for RMBP performance is presented in Table 3. 
Table 3. Physical Performance Results

\begin{tabular}{|c|c|c|c|}
\hline Time & Control & Supplement & Overall \\
\hline \multicolumn{4}{|c|}{ Vertical Jump (in) } \\
\hline Pre & $23.8 \pm 3.4$ & $23.5 \pm 4.7$ & $23.7 \pm 4.0$ \\
\hline Post & $23.9 \pm 3.8$ & $23.7 \pm 4.6$ & $23.8 \pm 4.1$ \\
\hline \multicolumn{4}{|c|}{ One Repetition Maximum Back Squat (lbs) } \\
\hline Pre & $277.9 \pm 100.9$ & $286.2 \pm 96.4$ & $282.3 \pm 96.9$ \\
\hline Post & $288.9 \pm 102.3$ & $305.0 \pm 105.2$ & $297.5 \pm 102.4^{*}$ \\
\hline \multicolumn{5}{|c|}{ One Repetition Maximum Bench Press (lbs) } \\
\hline Pre & $215.3 \pm 60.8$ & $218.1 \pm 64.7$ & $216.8 \pm 61.9$ \\
\hline Post & $219.6 \pm 63.2$ & $222.8 \pm 65.2$ & $221.3 \pm 63.2^{*}$ \\
\hline \multicolumn{5}{|c|}{ Repetition Maximum Bench Press (reps) } \\
\hline Pre & $13.2 \pm 4.3$ & $11.7 \pm 4.1$ & $12.5 \pm 4.2$ \\
\hline Post & $12.5 \pm 3.1$ & $11.8 \pm 3.8$ & $12.1 \pm 3.4$ \\
\hline
\end{tabular}

* Indicates significant main effect for time $(p<0.05)$

There was no significant interaction or main effect of group for $\mathrm{VO}_{2 \text { Peak }}(p>.05)$. There was a significant main effect of time for $\mathrm{VO}_{2 \text { Peak }}(p=.001)$. The mean values for $\mathrm{VO}_{2 \text { Peak }}$ for both the placebo and supplemental group were significantly greater post-intervention than pre-intervention (Pre: $37.8 \pm 7.5 \mathrm{ml} / \mathrm{kg} / \mathrm{min}$; Post: $40.5 \pm$ $8.1 \mathrm{ml} / \mathrm{kg} / \mathrm{min}$ ). Data for $\mathrm{VO}_{2 \text { Peak }}$ is presented in Table 4 .

There was no significant interaction or main effect of group for RER $(p>.05)$. There was a significant main effect of time for RER $(p=.000)$. The mean values for RER for both the placebo and supplemental group were significantly greater post-intervention than pre-intervention (Pre: $1.09 \pm .06$; Post: $1.17 \pm .08$ ). Data for RER is presented in Table 4.

There were no significant interactions or main effects of time or group for $\mathrm{VE} / \mathrm{VO}_{2}(p>.05)$. There was no significant interaction or main effect of group for $\mathrm{VE} / \mathrm{VCO}_{2}(p>.05)$. There was a significant main effect of time for $\mathrm{VE} / \mathrm{VCO}_{2}(p=.000)$. The mean values for $\mathrm{VE} / \mathrm{VCO}_{2}$ for both the placebo and supplemental group were significantly greater pre-intervention than post-intervention (Pre: $30.8 \pm 5.0$; Post: $28.6 \pm 3.8$ ). Data for both $\mathrm{VE} / \mathrm{VO}_{2}$ and $\mathrm{VE} / \mathrm{VCO}_{2}$ are presented in Table 4.

Table 4. Cardiovascular Performance Results

\begin{tabular}{|c|c|c|c|}
\hline Time & Control & Supplement & Overall \\
\hline \multicolumn{4}{|c|}{$\mathrm{VO}_{2 \text { peak }}(\mathrm{ml} / \mathrm{kg} / \mathrm{min})$} \\
\hline Pre & $38.9 \pm 6.5$ & $37.1 \pm 8.2$ & $37.9 \pm 7.5$ \\
\hline Post & $41.1 \pm 8.0$ & $40.2 \pm 8.4$ & $40.5 \pm 8.1^{*}$ \\
\hline \multicolumn{4}{|c|}{ Respiratory Exchange Ratio } \\
\hline Pre & $1.11 \pm .07$ & $1.09 \pm .06$ & $1.09 \pm .06$ \\
\hline Post & $1.17 \pm .08$ & $1.17 \pm .08$ & $1.17 \pm .08^{*}$ \\
\hline \multicolumn{4}{|c|}{$\mathrm{VEO}_{2}$} \\
\hline Pre & $32.4 \pm 4.0$ & $35.0 \pm 7.6$ & $33.8 \pm 6.2$ \\
\hline Post & $32.9 \pm 3.9$ & $33.8 \pm 5.5$ & $33.4 \pm 4.8$ \\
\hline \multicolumn{4}{|c|}{$\mathrm{VE} / \mathrm{VCO}_{2}$} \\
\hline Pre & $29.5 \pm 3.5$ & $31.9 \pm 5.9$ & $30.8 \pm 5.0$ \\
\hline Post & $28.1 \pm 3.3$ & $28.9 \pm 4.3$ & $28.6 \pm 3.8$ \\
\hline
\end{tabular}

*: Indicates significant main effect for time $(p<0.05)$

There was no significant interaction or main effect of group for lactate threshold (LT; $p>.05$ ). Lactate threshold was identified as the point at which blood lactate concentration exceeded a concentration of $4.0 \mathrm{mmol} / \mathrm{L}$ [24, Nicolson \& Sleivert, 2001). There was a significant main effect of time for LT ( $p=.001)$. The mean values for LT for both the placebo and supplemental group were significantly greater post-intervention than pre-intervention (Pre: $26.5 \pm 5.3 \mathrm{ml} / \mathrm{kg} / \mathrm{min}$; Post: $28.3 \pm$ $5.7 \mathrm{ml} / \mathrm{kg} / \mathrm{min}$ ). Data for LT is presented in Table 5 .

There was no significant interaction or main effect of group for peak lactate concentration $(p>.05)$. There was a significant main effect of time for peak lactate concentration $(p=.002)$. The mean values for peak lactate concentration for both the placebo and supplemental group were significantly greater post-intervention than pre-intervention (Pre: $5.83 \pm 2.1 \mathrm{mmol} / \mathrm{L}$; Post: $9.43 \pm 3.3$ $\mathrm{mmol} / \mathrm{L}$ ). Data for peak Lactate concentration is presented in Table 5.

Table 5. Lactate Kinetics

\begin{tabular}{|c|c|c|c|}
\hline Time & Control & Supplement & Overall \\
\hline \multicolumn{4}{|c|}{ Lactate Threshold $(\mathrm{ml} / \mathrm{kg} / \mathrm{min})$} \\
\hline Pre & $27.2 \pm 4.5$ & $25.9 \pm 5.8$ & $26.5 \pm 5.3^{*}$ \\
\hline Post & $28.7 \pm 5.6$ & $28.1 \pm 5.9$ & $28.4 \pm 5.7$ \\
\hline \multicolumn{4}{|c|}{ Peak Lactate Concentration $(\mathrm{mmol} / \mathrm{L})$} \\
\hline Pre & $6.0 \pm 2.1$ & $5.5 \pm 2.1$ & $5.8 \pm 2.1^{*}$ \\
\hline Post & $9.5 \pm 3.7$ & $9.1 \pm 2.3$ & $9.4 \pm 3.3$ \\
\hline
\end{tabular}

*: Indicates significant main effect for time $(p<0.05)$

\section{Discussion}

The primary purpose of this research study was to investigate the effects of chronic ingestion of a proprietary beta alanine supplement on body composition, indices of strength and power performance, and markers of cardiovascular endurance in physically active college-aged males. Specifically, the researcher intended to assess the potential effects of beta alanine ingestion on performance of a variety of physical tests directly related to military performance standards in a group of students commissioning within the Air Force, Army, Navy and Marine Corps. As performance on these physical tests often means the difference between being offered a commission and being rejected from the selected branch of service, any potential advantage gained by supplementation could play a major role in determining the future of these cadets.

The researcher hypothesized that chronic supplement ingestion would result in improved body composition, enhanced indices of strength and power performance, and improvements in markers of cardiovascular endurance, specifically a delay in the onset of blood lactate accumulation, known in this investigation as the lactate threshold (LT). A major finding of this investigation was that the supplement conferred no statistically significant 
performance enhancement pertaining to any of the measured variables across both the control and experimental groups.

Previous research has demonstrated significant improvements in both body composition and fat-free mass following extended bouts of beta-alanine supplementation relative to a placebo [30, 19, 31, 32, 3]. Kern and Robinson [33] performed an investigation of the effects of 4 grams/day of beta-alanine for a period of 8 weeks on body composition in collegiate football players and wrestlers. The researchers noted an average increase in fat-free mass of $2.1 \pm 3.6 \mathrm{lbs}$, increasing from $180.6 \mathrm{lbs}$ to $182.6 \mathrm{lbs}$ for the supplement group, and a slight increase of $1.1 \pm 2.3 \mathrm{lbs}$, increasing $169.5 \mathrm{lbs}$ to $170.6 \mathrm{lbs}$ for the placebo group. Additionally, the supplemental group declined only $0.1 \pm$ $1.1 \%$, dropping from $10.6 \%$ to $10.5 \%$, relative to the placebo group, whose body fat values increased $0.8 \pm 1.5 \%$, from $9.5 \%$ to $10.3 \%$ over the course of the investigation. In the current investigation, the supplement group dropped approximately $0.1 \pm 2.1 \mathrm{lbs}$ of lean body mass, going from $151 \mathrm{lbs}$ to $150.9 \mathrm{lbs}$, and losing $0.3 \pm 1.1 \%$ of body fat, going from $14.8 \%$ to $14.5 \%$. The placebo group gained approximately $2.5 \pm 1.8 \mathrm{lbs}$ of lean body mass, going from $154.9 \mathrm{lbs}$ to $157.4 \mathrm{lbs}$, and dropping $1.0 \pm 1.3 \%$ body fat, going from 15.9 to $14.9 \%$. While these values are not statistically significant, body composition has become a more prevalent part of the selection criteria associated with awarding competitive ROTC scholarships. The difference between getting accepted and rejected has become a matter of mere percentage points [33].

When consumed over the course of 2 to 10 weeks, and from 1.6 to $6.4 \mathrm{~g} /$ day, beta-alanine supplementation results in statistically significant increases in intramuscular carnosine content, improved physical performance, and demonstrable enhancements in body composition [29, 34]. By augmenting muscle carnosine concentration, beta alanine consumption will enhance the ability of an individual to perform exercise tasks that may result in acidosis of the muscle. However, not all individuals will garner as great a benefit from supplementation, as pre-existing intramuscular carnosine concentrations will determine the effectiveness of additional supplementation $[35,36]$. Though this investigation fell within the aforementioned range of both time and quantity, lasting for 6 weeks and requiring consumption of $6 \mathrm{~g} / \mathrm{day}$ of supplement or placebo, no statistically significant changes were noted.

Beta-alanine has been identified by many researchers as the rate-limiting factor in intramuscular carnosine concentration, meaning that without this beta-amino acid, individuals would be deficient in carnosine and in turn they would no longer be able to adequately maintain homeostasis at the intracellular level during physical exertion $[23,22,17,19,24]$. Researchers therefore have supported the notion that extraneous beta-alanine supplementation would augment carnosine synthesis, allowing individuals to better buffer intracellular Hydrogen ions, permitting sustained efforts for greater periods of time at higher exercise intensities [23, 19]. Specifically, beta alanine ingestion may enhance performance of activities relying heavily on anaerobic glycolysis $[13,16$, $14,15,21,19]$.

Unlike the majority of the previous research, the major finding of this investigation was that while there were significant improvements in performance for most all strength and cardiovascular performance parameters when comparing pre- to post-testing attributed to the learning effect associated with repeated activity performance, there were no significant differences between the placebo and supplemental group. Previous research has demonstrated significant improvements in activities of both short and long duration, ranging from resistance training [32] the $100-800 \mathrm{~m}$ run in track $[13,14]$, Greco-Roman wrestling [15], certain military fitness assessments across all branches of the services [16, 19], and long duration endurance activities, including the $3,000 \mathrm{~m}$ and the $10,000 \mathrm{~m}$ run [21].

Jordan et al [24] sought to identify the effects of beta-alanine supplementation on lactate threshold. Utilizing a double-blind, placebo-controlled experimental design, subjects consumed either $6 \mathrm{~g} /$ day of beta alanine or maltodextrin (placebo). From the results, the researchers noted a significant increase in the $\mathrm{VO}_{2 \text { peak }}$ at which the lactate threshold (LT) occurred, improving from $40.56 \pm$ $6.1 \mathrm{ml} / \mathrm{kg} / \mathrm{min}$ to $41.58 \pm 6.2 \mathrm{ml} / \mathrm{kg} / \mathrm{min}$. The placebo group demonstrated only a slight improvement in percentage of $\mathrm{VO}_{2 \text { peak }}$ at which LT was attained, going up from $36.65 \pm$ $5.2 \mathrm{ml} / \mathrm{kg} / \mathrm{min}$ to $38.41 \pm 5.1 \mathrm{ml} / \mathrm{kg} / \mathrm{min}$. This improvement corroborates the findings of the current investigation, which demonstrated a significant increase in LT from 25.9 $\pm 5.8 \mathrm{ml} / \mathrm{kg} / \mathrm{min}$ to $28.1 \pm 5.9 \mathrm{ml} / \mathrm{kg} / \mathrm{min}$. However, unlike with Jordan et al [24], the delayed onset of LT in the supplemental group was nearly identical in the placebo group as well $(27.2 \pm 4.5 \mathrm{ml} / \mathrm{kg} / \mathrm{min}$ to $28.7 \pm 5.6$ $\mathrm{ml} / \mathrm{kg} / \mathrm{min}$ ). Additionally, the $\mathrm{VO}_{2 \text { peak }}$ levels were lower in the current investigation as compared to the literature, suggesting a compounded effect of beta-alanine supplementation associated with greater cardiovascular fitness levels.

All subjects had at least 2 years of experience in both high-intensity resistance training, as well as cardiovascular endurance training. Activity records indicated that regular exercise regimens included moderate to maximal-effort total body weight lifting, sprinting, plyometric exercises, and regular endurance training. As the subjects were accustomed to the cardiovascular stimuli of both resistance exercise and variable intensity endurance training, they may have previously elevated intramuscular carnosine levels. This elevation will have a substantial moderating effect towards the potential benefits of supplemental beta-alanine [29].

Hoffman et al [19] investigated the effects of 30-days of beta-alanine ingestion on a series of military-specific tasks, 
ranging from short duration, high intensity (50-m casualty carry), to long duration, endurance exercise ( $2.5 \mathrm{~km}$ run). Using a double blind protocol, the researchers noted no significant changes in the $2.5 \mathrm{~km}$ run, 1 -min sprint, repeated $30-\mathrm{m}$ sprint, and target shooting activity. However, the researchers identified a significant improvement in $50-\mathrm{m}$ casualty carry. Hoffman et al [19] emphasized the importance of maximum strength and power as major contributing factors to the performance of the $50-\mathrm{m}$ casualty carry. In the current investigation, subjects in both the control and supplemental group demonstrated significant improvements in performance of the 1-repetition maximum back squat $(277.9 \pm 100.9 \mathrm{lbs}$ to $288.9 \pm 102.3 \mathrm{lbs}$ and $286.2 \pm 96.4 \mathrm{lbs}$ to $305.0 \pm 105.2 \mathrm{lbs}$, respectively) and bench press $(215.3 \pm 60.8 \mathrm{lbs}$ to $219.6 \pm$ $63.2 \mathrm{lbs}$ and $218.1 \pm 64.7 \mathrm{lbs}$ and $222.8 \pm 65.2 \mathrm{lbs}$, respectively). While there were no statistically significant differences, the values attained for supplement and placebo groups, the supplemental group demonstrated a slightly greater increase in 1-repetition back squat (19 lbs vs. 11 lbs), which could potentially translate into greater performance of this military-specific task.

Additional discordant findings may be explained by a few factors associated with investigative shortcomings and environmental constraints. Unlike with previous research, baseline carnosine concentration was not evaluated, which was a major limitation [29]. Additionally, much of the previous research was conducted using sedentary and moderately fit individuals $[36,30]$. This investigation used a novel group of highly trained and motivated individuals.

Another potential cause for discordance was that while asked to refrain from consuming additional dietary supplements over the duration of the investigation, subjects may have not maintained strict compliance throughout the entirety of the investigation. Secondly, technical issues arose on occasion with unanticipated equipment failure. Finally, there was a dramatic rate of attrition for this investigation. At the onset, the investigation had over 60 participants. With $50 \%$ of the subject population withdrawing over the course of the 6-week intervention, it is possible that this data may have simply moderated or potentially amplified any potential trends in changes associated with beta-alanine supplementation.

\section{Conclusions}

In conclusion, beta-alanine demonstrated no significant advantage over placebo relative to changes in body composition, strength, power, and cardiovascular performance parameters. These findings contradict much of the current literature. Unfortunately, many of the previously mentioned limiting factors, including current intramuscular carnosine levels, may have played a major role in hindering potential statistically measurable outcomes. Further research is needed to better understand the potential benefits that beta-alanine may play in enhancing physical performance in this unique population. Implementing better control factors through stricter subject selection, classifying subjects based upon current intramuscular carnosine levels, and regulating dietary composition are all factors that may play a major role in altering potential future outcomes.

\section{Acknowledgements}

We are very grateful to all of the subjects for their willingness to readily participate in this investigation. We would also like to express our gratitude to the Department of Physical Education and Virginia Military Institute and the Department of Physical Education and the United States Military Academy at West Point for their guidance and assistance throughout this process.

\section{REFERENCES}

[1] R. G. Lockie, A. Murphy, A. Schultz, T. Knight, X. Janse de Jonge. The Effects of Different Speed Training Protocols on Sprint Acceleration Kinematics and Muscle Strength and Power in Field Sports Athletes. Journal of Strength and Conditioning Research, Vol. 26, No. 6, 1539-1550. 2012.

[2] U. Wisloff, C. Castagna, J. Helgerud, R. Jones, J. Hoff. Strong Correlation of Maximal Squat Strength with Sprint Performance and Vertical Jump Height in Elite Soccer Players. British Journal of Sports Medicine, Vol. 38, No. 3, 215-228. 2004.

[3] T. Platanou, E. Varamenti. Relationships between Anthropometric and Physiological Characteristics with Throwing Velocity and on Water Jump of Female Water Polo Players. The Journal of Sports Medicine and Physical Fitness, Vol. 51, No. 2, 185-193. 2011.

[4] R. Silvestre, C. West, C. Maresh, W. Kraemer. Body Composition and Physical Performance in Men's Soccer: A Study of a National Collegiate Athletic Association Division I Team. Journal of Strength and Conditioning Research, Vol. 20, No. 1, 177-183. 2006.

[5] G. Sporis, I. Jukic, D. Bok, D. Vuleta, D. Harasin. Impact of Body Composition on Performance in Fitness Tests among Personnel of the Croatian Navy. Collegium Anthropologicum, Vol. 35, No. 2, 335-339. 2011.

[6] P. Aagaard. Making Muscles "Stronger": Exercise, Nutrition, Drugs. Journal of Musculoskeletal and Neuronal Interactions, Vol. 4, No. 2, 165-174. 2004.

[7] J. L. Andersen, P. Aagaard. Effects of Strength Training on Muscle Fiber Types and Size: Consequences for Athletes Training for High-Intensity Sport [Supplemental Material]. Scandinavian Journal of Medicine and Science in Sports, Vol. 20, No. 2, 32-38. 2010.

[8] W. L. Kenney, J.H. Wilmore, D. L. Costill. Physiology of 
Sport and Exercise, $5^{\text {th }}$ Edition. Champaign, IL: Human Kinetics. 2010

[9] K. S. Saladin. Anatomy and Physiology, $3^{\text {rd }}$ Edition. New York: McGraw Hill Company. 2004.

[10] P. O. Astrand, K. Rodahl, H. A. Dahl, S. B. Stromme. Textbook of Work Physiology: Physiological Bases of Exercise, $4^{\text {th }}$ Edition. Champaigh, IL: Human Kinetics. 2003

[11] L. E. Armstrong. Exertional Heat Illnesses. Champaign, IL: Human Kinetics Publishers, Inc. 2003.

[12] G. Brooks, T. Fahey, K. Baldwin. Exercise Physiology: Human Bioenergetics, $4^{\text {th }}$ Edition. New York, NY: McGraw-Hill Inc. 2005.

[13] C. Bret, L. Messonnier, J. M. N. Nouck, A. B. Dufour, J. R. Lacour. Differences in Lactate Exchange and Removal Abilities in Athletes Specialized in Different Track Running Events (100 to $1500 \mathrm{~m}$ ). International Journal of Sports Medicine, Vol. 24, No. 2, 108-113. 2003.

[14] S. Gupta, A. Goswami, S. Mukhopadhyay. Heart Rate and Blood Lactate in 400m Flat and 400m Hurdle Running: A Comparative Study. Indian Journal of Physiology and Pharmacology, Vol. 42, No. 3, 361-366. 1999.

[15] H. Karnincic, Z. Tocilj, O. Uljevic, M. Erceg. Lactate Profile during Greco-Roman Wrestling Match. Journal of Sports Science and Medicine, Vol. 8, No. CSSI3, 17-19. 2009.

[16] J. S. Cuddy, D. R. Slivka, W. S. Hailes, B. C. Ruby. Factors of Trainability and Predictability Associated with Military Physical Fitness Test Success. Journal of Strength and Conditioning, Vol. 25, No. 12, 3486-3494. 2011.

[17] J. R. Hoffman, G. Landau, J. R. Stout, M. Dabora, D. S. Moran, N. Sarvit, I. Ostfeld. B-Alanine Supplementation Improves Tactical Performance But Not Cognitive Function in Combat Soldiers, Journal of the International Society of Sports Nutrition, Vol. 11, 1-15. 2014.

[18] J. R. Hoffman, G. Landau, J. R. Stout, M. W. Hoffman, N. Shavit, P. Rosen, I. Ostfeld. B-Alanine Ingestion Increases Muscle Carnosine Content and Combat Specific Performance in Soldiers. Amino Acids, Vol. 47, 627-636. 2015.

[19] B. Sperlich, M. Krueger, C. Zinner, S. Achtzehn, M. de Marees, J. Mester. Oxygen Uptake, Velocity and Lactate Threshold, and Running Economy in Elite Special Forces. Military Medicine, Vol. 176, No. 2, 218-221. 2011.

[20] H. G. Simoes, B. S. Denadai, V. Baldiserra, C. S. Campbell, D. W. Hill. Relationships and Significance of Lactate Minimum, Critical Velocity, heart Rate Deflection and $3000 \mathrm{~m}$ Track-Tests for Running. Journal of Sports Medicine and Physical Fitness, Vol. 45, No. 4, 441-451. 2005 .

[21] C. Sale, C. A. Hill, J. Ponte, R. C. Harris. Beta-Alanine Supplementation Improves Endurance of the Knee extensor Muscles. Journal of the International Society of Sports Nutrition, Vol. 9, No. 26, 1-7. 2012.

[22] J. Caruso, J. Charles, K. Unruh, R. Giebel, W. Potter. Ergogenic Effects of Beta-Alanine and Carnosine: Proposed Future Research to Quantify Their Efficacy.
Nutrients, Vol. 4, 585-601. 2012.

[23] R. C. Harris, J. A. Wise, K. A. Price, H. J. Kim, C. K. Kim, C. Sale. Determinants of Muscle Carnosine Content. Amino Acids, Vol. 53, 5-12. 2012.

[24] P. Bellinger. Beta-Alanine Supplementation for Athletic Performance: An Update. Journal of Strength and Conditioning Research. Vol. 28, No. 6, 1-36. 2014.

[25] T. Jordan, J. Lukaszuk, M. Misic, J. Umoren. Effect of Beta-Alanine Supplementation on the Onset of Blood Lactate Accumulation (OBLA) During Treadmill Running: Pre/Post 2 Treatment Experimental Design. Journal of the International Society of Sports Nutrition, Vol. 7, No. 20, 1-7. 2010.

[26] R. A. Oppliger, C. Bartok. Hydration Testing of Athletes. Sports Medicine, Vol. 32, No. 15, 959-971. 2002.

[27] E. F. Coyle, A. R. Coggan, M. K. Hopper, T. J. Walters. Determinants of Endurance in Well-Trained Cyclists. Journal of Applied Physiology, 64, 2622-2630. 1988.

[28] R. W. Pettitt, I. E. Clark, S. M. Ebner, D. T. Sedgeman, S. R. Murray. Gas Exchange Threshold and VO2max Testing for Athletes: An Update. Journal of Strength \& Conditioning Research, Vol. 27, No. 2, 549-555. 2013.

[29] T. R. Baechle, R. W. Earle, D. Wathen. Resistance Training. In: T. R. Baechle, R. W. Wathen (eds.) Essentials of Strength Training and Conditioning, $3^{\text {rd }}$ Edition. Champaign, IL: Human Kinetics.

[30] A. Baguet, J. Bourgois, L. Vanhee, E. Achten, W. Derave. Important Role of Muscle Carnosine in Rowing Performance. Journal of Applied Physiology, Vol. 109, 1096-1101. 2010.

[31] R. M. Hobson, B. Saunders, G. Ball, R. C. Harris, C. Sale. Effects of Beta-Alanine Supplementation on Exercise Performance: A Meta-Analysis. Amino Acids, Vol. 43, 25-37. 2012.

[32] R. P. Lowery, J. M. Joy, J. E. Dudeck, E. O. de Souza, S. A. McCleary, S. Wells. Effects of 8 weeks of Expand $\AA 2 x$ Pre Workout Supplementation on Skeletal Muscle Hypertrophy, Lean Body Mass, and Strength in Resistance Trained Males. Journal of the International Society of Sports Nutrition, Vol. 10, No. 44, 1-6. 2013.

[33] B. D. Kern, T. L. Robinson. Effects of Beta-Alanine Supplementation on Performance and Body Composition in Collegiate Wrestlers and Football Players. Journal of Strength and Conditioning Research, Vol. 25, No. 7, 1804-1815. 2011.

[34] A. P. Crombie, P. Y. Liu, M. J. Ormsbee, J. Z. Ilich. Weight and Body-Composition Change During the College Freshman Year in Male General-Population Students and Army Reserve Officer Training Corps (ROTC) Cadets. International Journal of Sports Nutrition and Exercise Medicine, Vol. 22, No. 6, 412-422. 2012.

[35] W. Derave, M. S. Ozdemir, R. C. Harris, A. Pottier, H. Reyngoudt, K. Koppo. Beta-Alanine Supplementation Augments Muscle Carnosine Content and Attenuates Fatigue during Repeated Isokinetic Contraction Bouts in Trained Sprinters. Journal of Applied Physiology, Vol. 103, 1736-1743. 2007. 
[36] P. L. Invernizzi, S. Benedini, S. Saronni, G. Merati, A. Bosio. The Acute Administration of Carnosine and Beta-Alanine Does Not Improve Running Anaerobic Performance and Has No Effect on the Metabolic Response to Exercise. Advances in Physical Education, Vol. 3, No. 4, 169-174. 2013.
[37] T. Bex, W. Chung, A. Baguet, S. Stegen, J. Stautemas, E. Achten, W. Derave. Muscle Carnosine Loading by Beta-Alanine Supplementation is More Pronounced in Trained vs. Untrained Muscles. Journal of Applied Physiology, Vol. 116, 204-209. 2014. 